\title{
INVESTIGATION OF THE ABSORPTION OF ANNIHILATION QUANTA IN SOLID AND LIQUID Ga AND BiSn ALLOY
}

\author{
A. KLuza ${ }^{a, *}$, W. Rudzińska ${ }^{b}$ And M. Szuszkiewicz ${ }^{b}$ \\ ${ }^{a}$ Institute of Experimental Physics, Wrocław University \\ Pl. M. Borna 9, 50-204 Wrocław, Poland \\ ${ }^{b}$ Institute of Physics, Opole University, Oleska 48, 45-052 Opole, Poland
}

\begin{abstract}
Absorption of positron annihilation quanta in $\mathrm{Ga}$ and $\mathrm{Bi}_{40} \mathrm{Sn}_{60}$ alloy in the solid and the liquid phase was investigated. It was found that the mechanism of absorption of annihilation quanta changes on passing from the solid to the liquid state. Results of measurements for $\mathrm{Ga}$ and $\mathrm{Bi}_{40} \mathrm{Sn}_{60}$ alloy show that for metals changing their density at the melting point the photopeak profiles are modified: their centers of gravity in liquid phase are displaced with respect to those in the solid phase and the number of counts under the photopeak changes, too.
\end{abstract}

PACS numbers: $64.70 . \mathrm{Dv}$

\section{Introduction}

For many years the positron annihilation spectroscopy has been successfully used for studying the structure and properties of metallic materials. Among others, results of numerous investigations of the temperature dependence of various annihilation parameters: $F(T)$ in angular correlation $(\mathrm{AC}), S(T)$ in the Doppler broadening (DB) and the mean positron lifetime $\tau(T)$ in positron lifetime spectra (PLS) method in a wide temperature range including the melting point were reported. An inspection of these results shows that, as a rule, the annihilation parameters mentioned above, increase on passing from the solid to the liquid state. For example, Triftshäuser [1] reported a $2 \%$ increase in the value of the $F(T)$ parameter at the melting point for In. Also Rudzińska et al. [2] made some investigations for In and observed about a $1 \%$ increase in the $F(T)$ parameter at $T_{\mathrm{m}}$. The specific volume of In increases on melting by about $2.7 \%$ and this is why the authors of the papers [1,2] interpreted an increase in the $F$ parameter at $T_{\mathrm{m}}$ as a result of the specific volume increase and/or as an effect of a change in the concentration and a type of positron trapping centers. This is an important problem

*corresponding author; e-mail: kluza@max.ifd.uni.wroc.pl 
because of the lack of a satisfactory theoretical model of positron annihilation in the liquid state — one does not know either the annihilation mechanism or the type of the positron trapping centers.

Experimental results for $\mathrm{Ga}$ indicate that the increase in the annihilation parameters at $T_{\mathrm{m}}$ is not only an effect of the specific volume change. The specific volume of $\mathrm{Ga}$ decreases at the melting point by about $3.4 \%[3]$, so one should expect a decrease in the values of the annihilation parameters on passing from the solid to the liquid state, but it was found that the mean positron lifetime for $\mathrm{Ga}$ increases from $160 \mathrm{ps}$ in the solid state to $260 \mathrm{ps}$ in the liquid state [4], and the results of $\mathrm{AC}$ measurements on $\mathrm{Ga}[5]$ show a $16 \%$ increase in the $F$ parameter at $T_{\mathrm{m}}$. Similar changes of the annihilation parameters values (but somewhat smaller) were observed for $\mathrm{Sn}[2,6]$.

Basing on these results, Chabik et al. [7] interpreted the observed changes in the annihilation parameters as an effect of the change in the absorption of the annihilation quanta. These authors performed the measurements of the temperature dependence of the peak counting rate $(\mathrm{AC})$ and observed an increase in the $F(T)$ parameter value on melting amounting $0.6 \%$ for Sn and $1 \%$ for In. They also made measurements of the amplitude spectra for $\mathrm{In}, \mathrm{Sn}$, and $\mathrm{Bi}$ in the solid and liquid state and found that: (1) after melting of In and Sn the area of $0.511 \mathrm{MeV}$ and $1.28 \mathrm{MeV}$ photopeaks significantly decreased, (2) the profiles of photopeaks in the spectrum measured for In and Sn were modified - their centers of gravity were shifted towards higher energies by about $3 \mathrm{eV}$. Exactly opposite effects were observed for $\mathrm{Bi}$, the specific volume of which, in contrast to In and $\mathrm{Sn}$, decreased on melting.

Not only pure metals but also their alloys are often investigated by the positron annihilation methods. Chabik et al. [8] performed measurements of the peak coincidence counting rate ( $F$ parameter) for $\mathrm{Bi}_{40} \mathrm{Sn}_{60}$ alloy in the function of temperature in the temperature interval ranging from room temperature to $400^{\circ} \mathrm{C}$ in three consecutive heating and cooling runs. They concluded that: (1) in the solid state the positrons are trapped mainly at the grain boundaries and above the solidus temperature the most active trapping centers are located in the volume of grains, (2) rapid changes of the $F$ parameter at $T_{\mathrm{m}}$ are correlated with changes of the volume and structure of $\mathrm{Bi}$ and $\mathrm{Sn}$ grains, (3) on heating the liquid a decay of positron trapping defects is observed, which is irreproducible in the cooling process, (4) the thermal history of the samples strongly affects the temperature dependence of the peak counting rate.

In the present work the investigations of the absorption of annihilation quanta in both the liquid and solid phases of $\mathrm{Ga}$ and $\mathrm{Bi}_{40} \mathrm{Sn}_{60}$ alloy were undertaken in order to check if the melting affects the absorption spectra and if the obtained results depend on the thermal history of the investigated sample.

\section{Experiment}

The scheme of the measuring system is shown in Fig. 1. The ${ }^{22} \mathrm{Na}$ source that was used emits $\gamma$ photons of the energy of $0.511 \mathrm{MeV}$ (the annihilation quantum) and $1.28 \mathrm{MeV}$ (the nuclear quantum). After passing through the absorbent, 
the $\gamma$ photons were detected by a $\mathrm{NaI}(\mathrm{Tl})$ detector and registered in a multichannel amplitude analyzer. The tested samples were placed in the measuring vessel, enabling the measurements in both the liquid and solid states of investigated materials. During the measurements of absorption spectra the temperature of a sample was stabilized and measured with an accuracy of $0.2^{\circ} \mathrm{C}$.

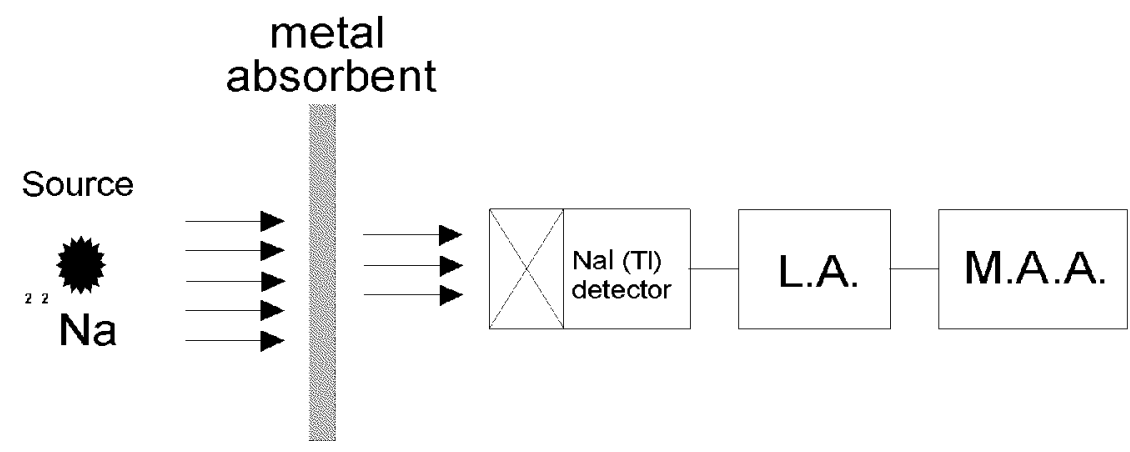

Fig. 1. Schematic diagram of the measuring system (L.A. - linear amplifier, M.A.A. - multichannel amplitude analyzer).

The powders of the investigated materials were placed in the measuring vessel and, after melting, heated up to the temperature slightly exceeding the melting point $\left(50^{\circ} \mathrm{C}\right.$ for $\mathrm{Ga}$ and $320^{\circ} \mathrm{C}$ for $\mathrm{Bi}_{40} \mathrm{Sn}_{60}$ alloy), at which the measurement of the amplitude spectrum for the liquid phase was carried out. Next, the samples were cooled to the room temperature and the measurement for the solid sample was made. In the case of $\mathrm{Bi}_{40} \mathrm{Sn}_{60}$ alloy the measurements were repeated in three consecutive heating/cooling runs for the same sample.

The measured amplitude spectra were not calibrated. Positions of the centroides (CTR) of the $0.511 \mathrm{MeV}$ and the $1.28 \mathrm{MeV}$ photopeaks were calculated by the SWAN program, according to the formula (1)

$$
\mathrm{CTR}=\frac{\sum_{i=1}^{n} Y_{i} k_{i}}{\sum_{i=1}^{n} k_{i}}
$$

where $k_{i}$ is the channel number, $Y_{i}$ - number of counts in the $i$-th channel, $n$ - the total number of all channels. Counts in all photopeak channels were summarized. The initial and the final channel of the photopeak were always the same. The accuracy of calculations of the CTR and of the sums of counts was about $0.05 \%$.

\section{Results and discussion}

The experimentally determined parameters of the photopeaks registered for the solid and liquid $\mathrm{Ga}$ are collected in Table I.

For a better visualisation of the differencies between the spectra measured for the liquid and solid $\mathrm{Ga}$, the spectrum for the solid $\left(20^{\circ} \mathrm{C}\right)$ was subtracted from the liquid state spectrum $\left(50^{\circ} \mathrm{C}\right)$. The resulting differences in the spectra are shown in Fig. 2. 
TABLE I

Parameters of photopeaks determined for the solid and liquid phase of Ga.

\begin{tabular}{l|c|c|c|c}
\hline \hline & \multicolumn{2}{|c|}{$0.511 \mathrm{MeV}$ photopeak } & \multicolumn{2}{c}{$1.28 \mathrm{MeV}$ photopeak } \\
\cline { 2 - 5 } & $\begin{array}{c}\text { CTR } \\
\text { (channel no.) }\end{array}$ & $\begin{array}{c}\text { sum of counts } \\
{\left[\times 10^{6}\right]}\end{array}$ & $\begin{array}{c}\text { CTR } \\
\text { (channel no.) }\end{array}$ & $\begin{array}{c}\text { sum of counts } \\
{\left[\times 10^{6}\right]}\end{array}$ \\
\hline Liquid Ga & 472 & $7.400 \pm 0.003$ & 1229 & $2.920 \pm 0.002$ \\
Solid Ga & 474 & $7.520 \pm 0.003$ & 1233 & $2.930 \pm 0.002$
\end{tabular}

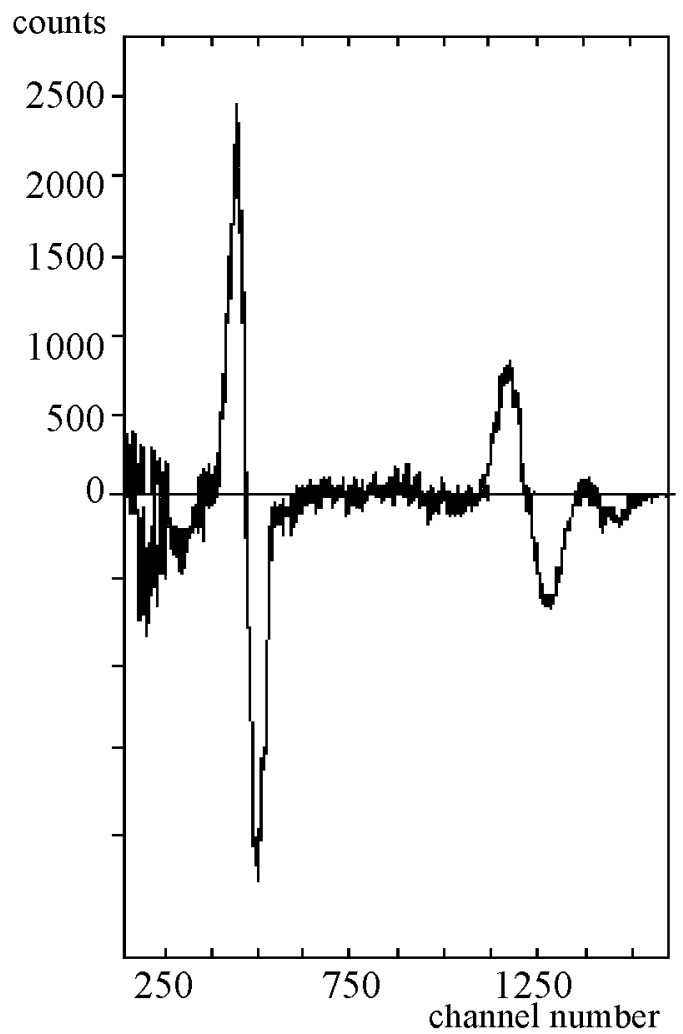

Fig. 2. Differences in the amplitude spectra for Ga.

The density of Ga increases on melting by $3.4 \%$ [8], so one can expect that the results obtained for $\mathrm{Ga}$ should be similar to the results reported for $\mathrm{Bi}[6]$, increasing its density on melting by $3.3 \%$ [3], and this was observed. Table I and Fig. 2 show that the profiles of the photopeaks change on passing from the solid to the liquid state: the CTRs for liquid phase are located at lower energies and the sums of counts are smaller than those for the solid phase. It should be stressed, however, that although the CTRs are displaced, the positions of the photopeak 
maximum for the solid and the liquid state are located in the same channel. The source of this effect is probably the change in the photoeffect and the Compton cross-sections associated with the change of the specific volume at $T_{\mathrm{m}}$. These results suggest that the changes in the $F, S$, or $\tau$ parameters observed in many works on positron annihilation and interpreted as yet, consistently with the vacancy model of the melting process, as a result of increase in a number of vacancies may be also caused by other factors, in particular by the change of the mechanism of the absorption, of the $\gamma$ photons.

The results of the measurements performed on the $\mathrm{Bi}_{40} \mathrm{Sn}_{60}$ alloy are presented in Table II and Figs. 3-5.

TABLE II

Parameters of photopeaks registered for liquid and solid phases of BiSn alloy determined in three consecutive heating/cooling cycles.



The composition of the investigated alloy was chosen intentionally. The density of $\mathrm{Bi}$ increases by about $3.3 \%$ and that of $\mathrm{Sn}$ decreases by about $2.7 \%$ on the melting [8]. Therefore, one could expect that the density of the $\mathrm{Bi}_{40} \mathrm{Sn}_{60}$ alloy should decrease on melting by $0.3 \%$ if only the assumption of the additivity (Vegard's rule) holds for this alloy. This way it could be possible to compare the results for the metallic material (Ga) showing a distinct change in the specific volume with those for a material showing almost no change of the specific volume on melting. As it follows from the data collected in Table I, the CTRs of the photopeaks corresponding to the energies of $0.511 \mathrm{MeV}$ and $1.28 \mathrm{MeV}$ for the liquid phase, determined in the first heating/cooling run, are shifted to higher energies, with respect to those for the solid state, and the sum of counts in case of each of the photopeaks for the liquid phase is higher than that for the solid state.

In each next heating/cooling cycle the differences between these quantities become smaller and smaller, and in the last cycle there is no difference between the solid and liquid state, which was expected. One can also see that each next spectrum shows lower number of registered $\gamma$ photons. These effects may be caused by the heating treatment that induces the homogenization of the alloy. The obtained results are consistent with the results of measurements of the temperature dependence of the $F$ parameter reported in [7]. 


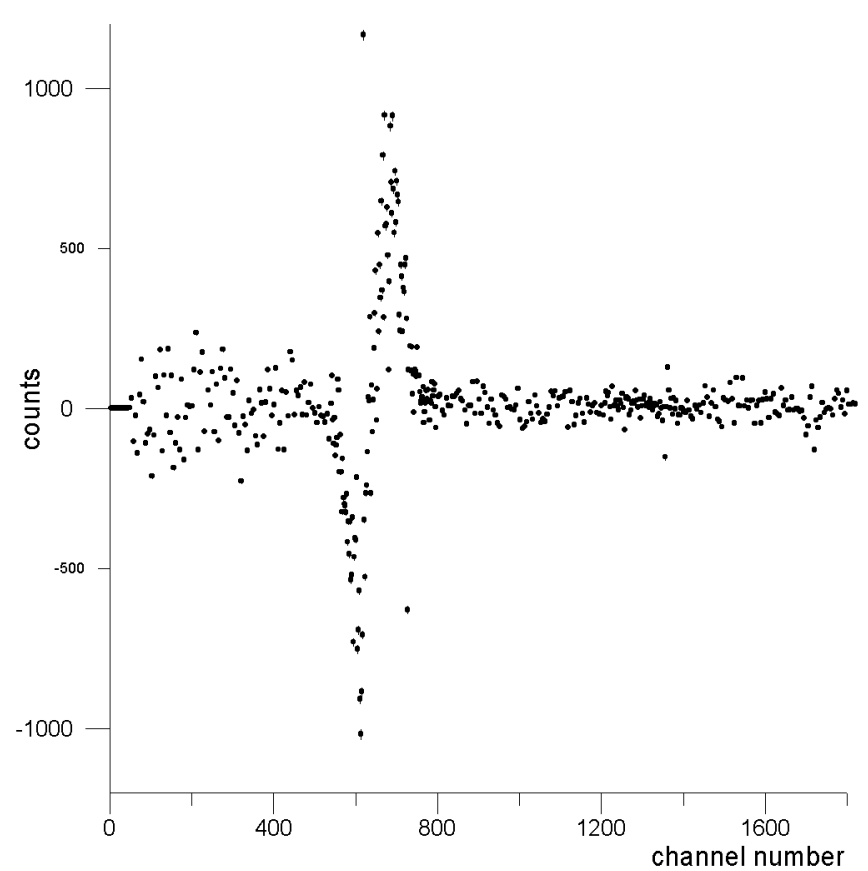

Fig. 3. Differences in the amplitude spectra for BiSn alloy determined in the 1st heating/cooling cycle.

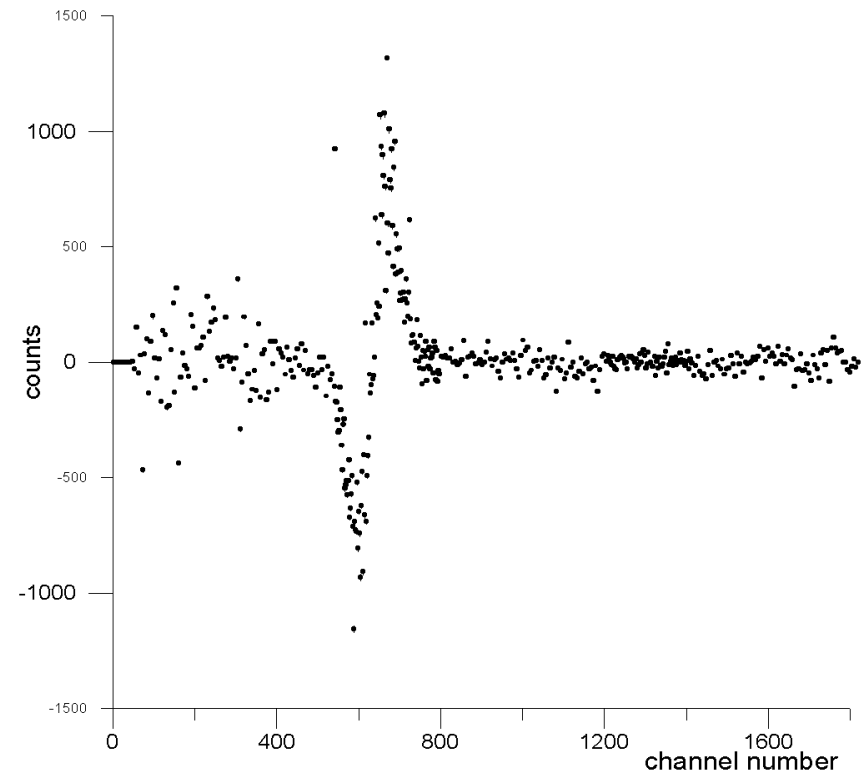

Fig. 4. Differences in the amplitude spectra for BiSn alloy determined in the 2nd heating/cooling cycle. 


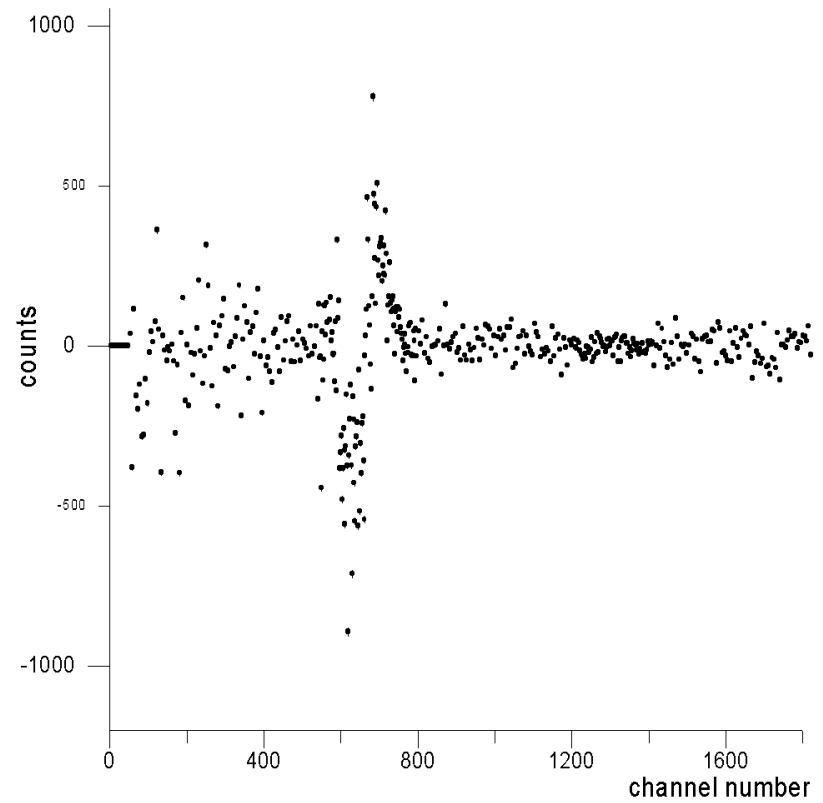

Fig. 5. Differences in the amplitude spectra for BiSn alloy determined in the 3rd heating/cooling cycle.

The results of the present study for Ga confirm the existence of a correlation between the density and the absorption spectra of the metal. The melting of the sample is associated with the change of the electron-to-atom bonding. The photoeffect proceeds with the atomic core electrons, whereas the Compton effect sets on the slightly bonded electrons. Therefore we may suggest that the source of the observed effects is the change in Compton cross-section associated with the change of the specific volume at the melting point.

\section{References}

[1] W. Triftshäuser, Phys. Rev. B 12, 4634 (1975).

[2] W. Rudzińska, S. Chabik, C. Szymański, Acta Univ. Wratislav. Mat. Fiz. Astron. 59, 137 (1991).

[3] M. Jeżewski, J. Kalisz, Tablice wielkości fizycznych, PWN, Warszawa 1957 (in Polish).

[4] W. Brandt, H.F. Waung, Phys. Lett. A 27, 700 (1968).

[5] C. Szymański, S. Chabik, J. Pająk, B. Rozenfeld, Phys. Status Solidi A 60, 375 (1980)

[6] N. Shah, A.L. Catz, Phys. Rev. B 30, 2498 (1984).

[7] S. Chabik, M. Szuszkiewicz, M. Szymański, in: Proc. 27th Seminar on Positron Annihilation, Jarnottówek (Poland) 1995.

[8] S. Chabik, W. Rudzińska, M. Szuszkiewicz, C. Szymański, Acta Phys. Pol. A 95, 479 (1999). 